\title{
In silico prediction of virus-derived small interfering RNAs and their putative host messenger targets in Solanum Iycopersicum infected by diffferent notato virus Y isolates
}

\author{
D. Catalano, F. Cillo, M. Finetti-Sialer \\ Istituto di Genetica Vegetale, Consiglio Nazionale delle Ricerche, Bari, Italy
}

\section{Motivations}

RNA silencing, or post-transcriptional gene silencing (PTGS), is a conserved mechanism in a broad range of eukaryotes. In plants, PTGS acts as an antiviral system and a successful virus infection requires suppression or evasion of the induced silencing response. Small interfering RNAs (siRNAs) accumulate in plants infected with RNA and DNA viruses and provide specificity to this RNAmediated immune system. High-throughput sequencing has contributed to expanding our previously knowledge of siRNA populations better describing their abundance, complexity and diversity in infected tissues. It is now known that siRNAs from virus-infected plants are extraordinarily abundant and diverse, and are widespread in near saturation at any region of either positive and negative genomic RNAs. However, certain regions of viral genomes ("hot spots") are usually more represented than others in sequenced siRNA populations. A gene involved in chlorophyll biosynthesis has been shown targeted by a siRNA derived from viral satellite RNA, revealing PTGS mechanism at basis of the symptom. Potato virus $Y(P V Y)$ is the type species of Potyvirus, a genus of agricultural importance belonging to the largest plant virus family, Potyviridae. The potyviral genome is a single-stranded, positive-sense RNA of ca. 10 kilobases (kb). PVYc-to and PVY-SON41 are two isolates of $\mathrm{PVY}$ that infect solanaceous hosts. While PVYc-to induces severe leaf distortion in different cultivars of tomato (Solanum lycopersicum), PVY-SON41 produces in the same host only a mild mosaic, followed by recovery. In order to elucidate the molecular mechanism underlying the different symptoms induced by PVYC-to and PVY-SON41 infections on tomato, we set up an in silico approach, mining genomic regions of $\mathrm{PVY}$ isolates and looking at possible RNA-based mechanisms where siRNA putatively generated from the PVY genome could target and suppress accumulation of host messenger RNA (mRNA), leading to dysfunctional biological processes that could explain different disease phenotypes.

\section{Methods}

A Perl script was used to extract the complete datasets of 21-mers from isolates PVYC-to and PVY-SON41 complete genomes, leading the scanning of the complete sequence, shifted by one base at the time. MySQL was used for identification of the 21-mers shared and unique between the two viral genomes, highlighting sequence differences that could be at the base of diverse induced symptoms. The data obtained in the previous step were used to build the genomic map of identical regions, by fancyGene. A BLAST analysis was conducted with the identified 21-mer dataset, considering only the dissimilar sequences between the two isolates (blastn-short identity > 94\%, max 2 mismatch or gap, alignment length $21 \mathrm{bp})$. The 21-mer were used as query and the tomato mRNA database (Solgenomics, release ITAG2.3) was used as target. The results were further used as input in a gene ontology analysis, through Blast2GO.

\section{Results}

Despite the high identity (>91\%) shared by the two viral genomes, the 21-mer dataset produced a high degree of variation, as the analyses showed 1750 identical and 9671 and 9680 dissimilar sets of 21-mer, for PVYc-to and PVYSON41, respectively. Data showed that 315 and 381 tomato mRNA were complementary to, and thus possible targets of, the in silico-generated siRNAs deduced from of PVYc-to and PVYSON41 sequences, respectively. The GO analysis showed different putative gene targets, involved in several metabolic pathways indicating specific targets for each isolate. Of particular interest, for instance, are putative target mRNAs of transcription factors with a known role concerning leaf development and symmetry. These genes, if differentially modulated during the infections of the two different PVY isolates, could cause the 
leaf malformations observed as major differ- have been selected from the in silico data for exential symptoms between the two PVY isolates. perimental validation in tomato plants mechaniTo validate the bioinformatic approach, target cally infected by virus isolates. genes with highly significant complementarity 